\title{
Wide-field quantitative imaging of tissue microstructure using sub-diffuse spatial frequency domain imaging: supplementary material
}

\author{
David M. McClatchy III ${ }^{1}$, Elizabeth J. Rizzo ${ }^{2}$, Wendy A. Wells ${ }^{2,3}$, Philip P. \\ Cheney $^{4}$, Jeeseong C. Hwang ${ }^{4}$, Keith D. Paulsen ${ }^{1,3}$, Brian W. Pogue ${ }^{1,3}$, \\ AND Stephen C. KaniCK ${ }^{1,3^{*}}$
}

${ }^{1}$ Thayer School of Engineering, Dartmouth College, 14 Engineering Drive, Hanover, NH 03755

${ }^{2}$ Department of Pathology, Dartmouth Hitchcock Medical Center, 1 Medical Center Drive, Lebanon, NH 03756

${ }^{3}$ Norris Cotton Cancer Center, Dartmouth Hitchcock Medical Center, 1 Medical Center Drive, Lebanon, NH 03756

${ }^{4}$ Quantum Elecromagnetics Division, National Institute of Standards and Technology, 325 Broadway Street, Boulder, CO 80305

Corresponding author: stephen.c.kanick@dartmouth.edu

Published 9 June 2016

\begin{abstract}
This document provides supporting content for the article "Wide-field quantitative imaging of tissue microstructure using sub-diffuse spatial frequency domain imaging," http:// dx.doi.org/10.1364/optica.3.000613. (c) 2016 Optical Society of America
\end{abstract}

http://dx.doi.org/10.1364/optica.3.000613.s001

\section{EXPANDED METHODS AND MATERIALS}

A. Sub-diffuse Spatial Frequency Domain Imaging Setup and Parameter Fitting

Imaging was performed using a commercial spatial frequency domain imaging system (Modulating Imaging, Inc., Irvine, CA). This unit acquired spatial frequencies over the range of $f_{x}=[0-0.2,0.5-0.9] \mathrm{mm}^{-1}$ in steps of $0.05 \mathrm{~mm}^{-1}$ with source LEDs centered about $[658,730,850] \mathrm{nm}$. In order to maximize signal from low-ordered scattered photons, cross-polarizing filters were removed. However, to mitigate the effect of specularly reflected photons, projections were obliquely illuminated and a signal acquired from a measurement of water in a deep $(\approx 50$ $\mathrm{cm}$ ) well with dark non-reflecting walls was subtracted from both the sample and reference intensity maps to approximate the specular reflection [1]. Maps of calibrated reflectance were calculated by ratioing demodulated intensity maps of the sample with a reference measurement of Intralipid $1 \%$ and multiplying by a model reflectance value calculated from Eqs. S1 \& S2 using well-documented optical properties for Intralipid [2]. The reflectance model is broken into piecewise contributions from a semi-empirical sub-diffusive expression, Eq. S1, described in Kanick et al. [3] and a diffusion theory model, Eq. S2, described in Cuccia et al. [4]. The combination is complementary because the sub-diffusive model, $R_{\text {SubDiff }}\left(f_{x}, \mu_{\mathrm{s}}^{\prime}, \gamma\right)$, is sensitive to scatter directionality but does not account for optical absorption whereas the diffusion theory model, $R_{\text {Diff }}\left(f_{x}, \mu_{\mathrm{a}}, \mu_{\mathrm{s}}^{\prime}\right)$, assumes diffusive photon propagation but characterizes both diffuse scatter and absorption. Thus, only spatial frequencies above $0.5 \mathrm{~mm}^{-1}$ [5], which have negligible signal contribution from absorption for typical optical properties of tissue in the near-infrared (NIR) window [6], were analyzed with the subdiffusive model, and spatial frequencies below $0.2 \mathrm{~mm}^{-1}$, which meet assumptions of diffusion for typical tissue optical properties in the NIR window, were analyzed with the diffusion theory model. The calibrated images of demodulated reflectance over the acquired spatial frequencies and wavelengths $R_{\mathrm{d}}\left(f_{x}, \lambda\right)$ were inverted into maps of $\mu_{\mathrm{s}}^{\prime}, \gamma$, and $\mu_{\mathrm{a}}$ with a non-linear least squares minimization using the expressions:

$$
\begin{aligned}
& R_{\text {SubDiff }}\left(f_{x}, \mu_{\mathrm{s}}^{\prime}, \gamma\right)= \\
& \eta\left(1+\left(\zeta_{4} \gamma^{-2}\right)\left(\mu_{s}^{\prime} f_{x}^{-1}\right)^{\left(-\zeta_{3} \gamma\right)}\right)\left[\frac{\left(\mu_{s}^{\prime} f_{x}^{-1}\right)^{\left(-\zeta_{2} \gamma\right)}}{\zeta_{1} \gamma^{2}+\left(\mu_{s}^{\prime} f_{x}^{-1}\right)^{\left(-\zeta_{2} \gamma\right)}}\right] \\
& R_{\text {Diff }}\left(f_{x}, \mu_{\mathrm{a}}, \mu_{\mathrm{s}}^{\prime}\right)= \\
& \frac{3 \alpha^{\prime} \frac{\mu_{\mathrm{s}}^{\prime}}{\mu_{\mathrm{tr}}}}{\left(\frac{\sqrt{3 \mu_{\mathrm{a}} \mu_{\mathrm{tr}}+\left(2 \pi f_{x}\right)^{2}}}{\mu_{\mathrm{tr}}}+1\right)\left(\frac{\sqrt{3 \mu_{\mathrm{a}} \mu_{\mathrm{tr}}+\left(2 \pi f_{x}\right)^{2}}}{\mu_{\mathrm{tr}}}+3 \alpha^{\prime}\right)}
\end{aligned}
$$




$$
R_{\mathrm{d}}\left(f_{x}, \mu_{\mathrm{a}}, \mu_{\mathrm{s}}^{\prime}, \gamma\right)= \begin{cases}R_{\text {Diff }} & \text { if } f_{x} \leq 0.2 \mathrm{~mm}^{-1} \\ R_{\text {SubDiff }} & \text { if } f_{x} \geq 0.5 \mathrm{~mm}^{-1}\end{cases}
$$

In the diffusive model, the parameter $\alpha^{\prime}$ is a proportionality constant, accounting for surface boundary effects [7]. In the subdiffusive model, the fitted model parameter set $[\eta, \zeta]$ reported in [3] provided accurate descriptions of reflectance intensities over a wide range of scattering properties, including: $\mu_{\mathrm{s}}^{\prime}=[0.3-10]$ $\mathrm{mm}^{-1}$ and $\gamma=[1.3-1.9]$. The present study utilized Monte Carlo (MC) simulations to characterize an expanded range of $\gamma$ values spanning a range that have been previously reported in tissue [3, 8-10]; this full range of $\gamma$ is not able to be considered using the Modified Henyey Greenstein phase function [11] that was used to develop Eqn. S1 in [3]. Here, a customized MC model was used to consider scattering phase functions calculated using Mie Theory, with input parameters used to specify a discrete set of scatterers in suspension. These simulations constructed phase functions with 7 discrete particle sizes in the range of $[0.1-2.0 \mu \mathrm{m}]$ with a fractal dimension of the scatterers in the range of [2.6-4.9] in steps of 0.1, with an index of refraction mismatch of $(1.37 / 1.33)$ between the scatterers and surrounding fluid; these input combinations yielded a set of 24 phase functions with a wide range of values for both $g_{1}=$ $[0.55-0.98]$ and $\gamma=[1.2-2.4]$. The MC simulations returned reflectance intensity vs. distance from a point source at an airmedium interface and a 1-dimensional Hankel transformation was used for conversion to a spatial frequency basis [4]. Values for $\mu_{s}^{\prime}$ and $f_{x}$ were selected to yield 40 values of dimensionless scattering (i.e. $f_{x} / \mu_{s}^{\prime}$ ) over the range [0 - 1] [-] for each sampled phase function. Figure S1 shows reflectance vs. dimensionless scattering for both $\mathrm{MC}$ simulations and predictions of Eqn. $\mathrm{S} 1$, in plots S1a and S1b respectively. The predictions of the semi-empirical model accurately match the simulated data, with the squared Pearson product moment correlation coefficient of $r^{2}=0.987$ and a mean absolute error between model and simulation data of $<9 \%$ over $f_{x} / \mu_{s}^{\prime}=[0-1]$ [-] for all simulated phase functions. Inspection of the data in Figure S1 highlight the non-uniqueness of reflectance intensity in terms of $\mu_{s}^{\prime}$ and $\gamma$; with reflectance monotonically decreasing with increasing $f_{x} / \mu_{s}^{\prime}$ and exhibiting a $\gamma$-specific proportionality for high spatial frequencies.

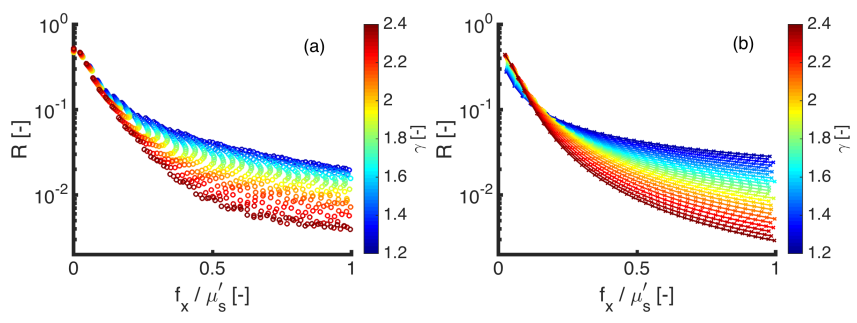

Fig. S1. Reflectance vs. dimensionless scattering from (a) Monte Carlo simulations and (b) semi-empirical model predictions.

Imaged $R_{d}\left(f_{x}, \lambda\right)$ data were fit to Eqs. S1 \& S2 on a pixelby-pixel basis, returning images of $\mu_{\mathrm{s}}^{\prime}, \gamma$, and $\mu_{\mathrm{a}}$ at each measured wavelength where $\mu_{\mathrm{s}}^{\prime}$ was assumed to follow a power law, $\mu_{\mathrm{s}}^{\prime}(\lambda)=A\left(\frac{\lambda}{800 \mathrm{~nm}}\right)^{-B}$, in which $A$ is the scatter amplitude and corresponds directly to $\mu_{\mathrm{s}}^{\prime}(800 \mathrm{~nm}), B$ is the scatter power, and $\gamma(\lambda)$ was fitted as a free parameter at each wavelength. An analysis algorithm was coded in MATLAB (R2015a, MathWorks Inc. Natick, MA) that automatically identified and excluded pixels that were non-scattering or contained specular reflection, and the code was deployed on an 8-core parallel processing CPU. The average time to recover a parameter map for a $2.5 \mathrm{~cm} \times 2.5$ $\mathrm{cm}$ tissue sample that contained roughly 3000 pixels was $\approx 1$ hour. Fitting time could be rapidly decreased by employing a look-up-table (LUT) and/or further parallelization of the fit procedure. All demodulated images were processed with a median filter having a kernel size of $2 \mathrm{~mm} \times 2 \mathrm{~mm}$ ([10 $\times 10]$ pixels) to reduce noise originating from a poor signal-to-noise ratio at higher spatial frequencies. Moreover, it is important to note that $97.5 \%$ of pixel-based estimates of $\gamma$ from tissue samples were within the validated parameter space for the semi-empirical model (i.e. $1.3<\gamma<2.4$ ), with the remaining $2.5 \%$ of pixels designated as outliers.

It is important to note that the model-based interpretation of high spatial frequency data in this study assumes statistical homogeneity in scatterer orientation. Previous work by Konecky et al. [12] showed that rotation of the illumination pattern could provide sensitivity to the micro-alignment and organization of scatterers within an imaged sample. Future work could consider rotation of the incident illumination to provide a coupled assessment of density, size, and orientation.

\section{B. Imaging of Sub-Diffuse Scattering Properties in the Pres- ence of a Strong Absorber}

An aqueous phantom set was constructed to confirm the accuracy of scattering parameters in the presence of absorption based attenuation. This phantom set contained nine phantoms with coupled variation of $D_{\mathrm{f}}=[3.6,4.1,4.6]$ and the absorption coefficient, which was varied by adding incremental amounts of Evans Blue dye to achieve $\mu_{\mathrm{a}}=[0.02-0.18] \mathrm{mm}^{-1}$ such that the ratio $\frac{\mu_{\mathrm{s}}^{\prime}}{\mu_{\mathrm{a}}}$ ranged from 5.6 to 50 at $658 \mathrm{~nm}$. Data in Fig. S2 show that both $\mu_{\mathrm{s}}^{\prime}$ and $\gamma$ are accurately recovered in the presence of a strong optical absorber.

\section{Heterogeneous Optical Property Phantoms: Step Change in Scatter Contrast}

While the aqueous polystyrene bead phantoms allow for the direct quantification of $\mu_{\mathrm{s}}^{\prime}$ and $\gamma$ with comparison to theoretical values from Mie theory, these phantoms fail to demonstrate the ability to spatially resolve scattering optical properties in a heterogeneous phantom. As such, agarose phantoms with a step change in either scatter size or density were constructed and imaged (I) to demonstrate proof of principle, and (II) to estimate resolution and sensitivity of the optical scattering parameters. Phantoms were gelled with $10 \mathrm{~g} / \mathrm{L}$ of agarose in phosphate buffered solution, which was brought to a boil, cooled to approximately $40 C^{\circ}$, mixed with the scattering agent, and finally poured into a rectangular mold submerged in ice for gelling to occur. For the step change in scatter size, $2 \mathrm{~g} / \mathrm{L}$ of titanium dioxide powder and $1.5 \%$ Intralipid were used as the scattering agents, while the step change in scatter density was $1 \%$ and 5 $\%$ Intralipid concentrations. A summary of the heterogeneous phantom data is shown in Figure S3. As shown in Figure S3 $(a, b)$, there is little visible contrast between the titanium dioxide and Intralipid phantoms and only slight contrast between the $1 \%$ and $5 \%$ Intralipid concentrations. However, the $\gamma$ and $\mu_{\mathrm{s}}^{\prime}$ optical property maps in Figure S3(c,d) very clearly recover scatter size and density based contrast. In Figure S3(e,f), the edge response 


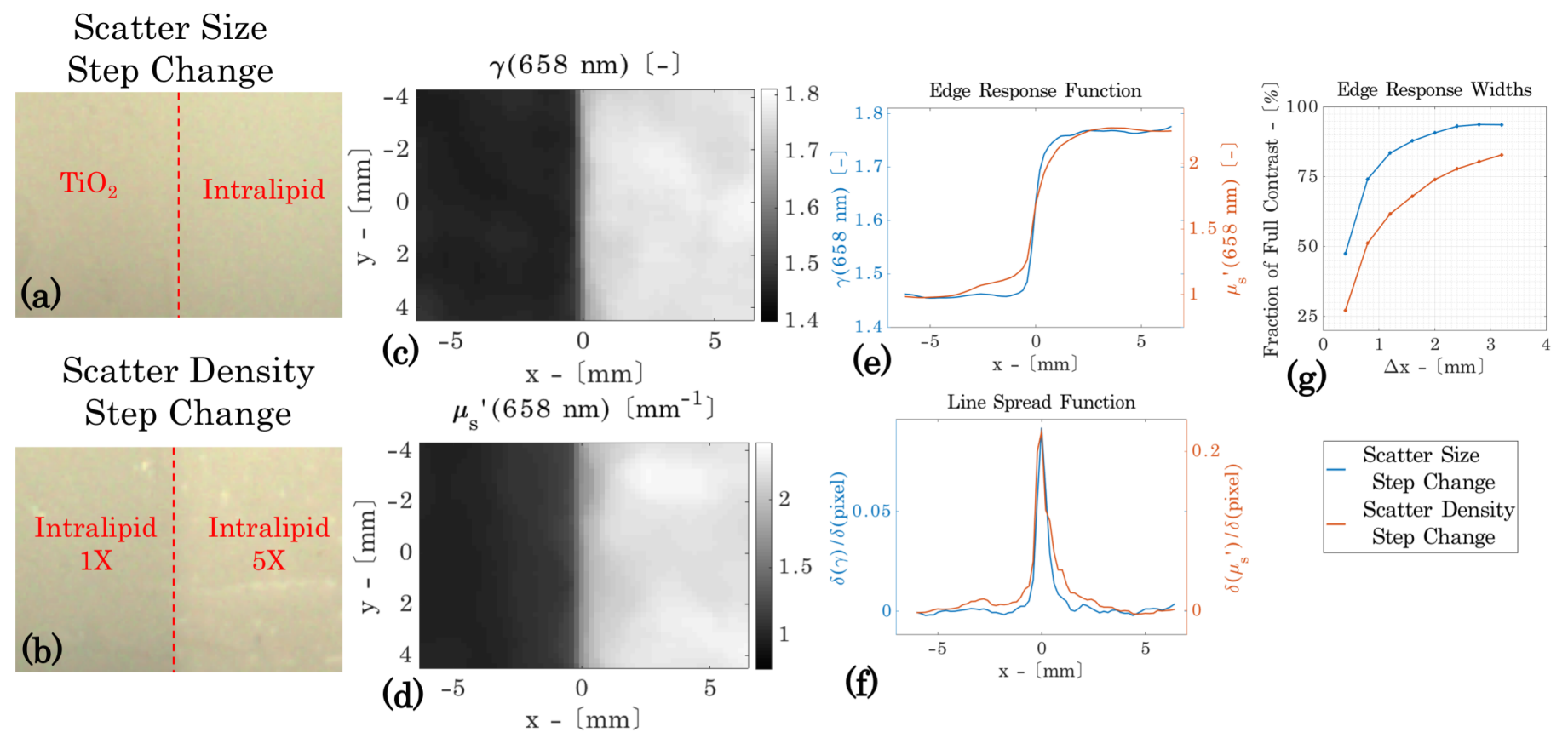

Fig. S3. Annotated photographs of scatter size (a) and density (b) step change agarose phantoms with titanium dioxide and Intralipid in (a) and 1\% and 5\% Intralipid in (b). Corresponding gray-scale optical property maps are shown, with $\gamma$ for scatter size change in (c) and $\mu_{\mathrm{s}}^{\prime}$ for scatter density change in (d). Edge response functions are shown in (e) for both $\gamma$ and $\mu_{\mathrm{s}}^{\prime}$ averaged in the y direction. Corresponding line spread functions are shown in (f), which is the derivative of (e) approximated by a forward finite difference. In $(\mathrm{g})$, the percentage of full contrast reached as a function of edge width across the step is plotted for both scatter size and density contrast. Note that a pixel width is $200 \mu \mathrm{m}$, and so in (g) each point is plotted in steps of $400 \mu \mathrm{m}$, iteratively adding a pixel to each side of the boundary.

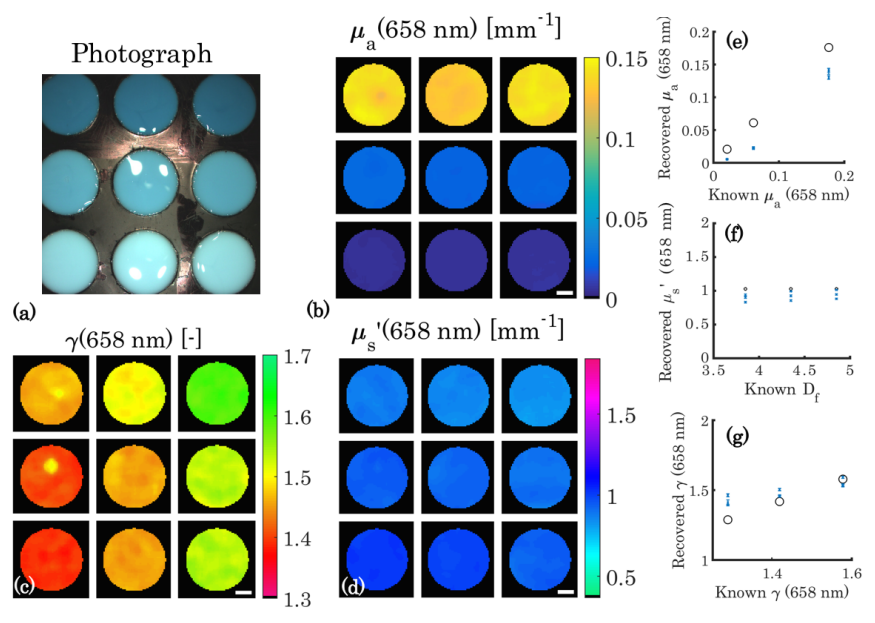

Fig. S2. (a) Photograph of the $\gamma$ and $\mu_{\mathrm{a}}$ variation phantom set, showing visible blue contrast between the various phantoms in each row with a change in $D_{\mathrm{f}}$ in each column. (b) Recovered $\mu_{\mathrm{a}}$, (c) $\gamma$, and (d) $\mu_{\mathrm{s}}^{\prime}$ maps at $658 \mathrm{~nm}$. (e-g) Reports recovered experimental mean values versus $D_{\mathrm{f}}$ for each well with error bars representing one standard deviation above and below the mean and black circles representing theoretical values for each optical property.

function and line spread function for $\gamma$ and $\mu_{\mathrm{s}}^{\prime}$ shows the spatial resolution and sensitivity of a step change of known contrast. The percentage of full contrast as a function of distance across the boundary is shown in Figure S3(g). The distance over which
$75 \%$ contrast is reached is approximately $800 \mu \mathrm{m}$ (4 pixels) for $\gamma$ and $2000 \mu \mathrm{m}$ (10 pixels) for $\mu_{\mathrm{s}}^{\prime}$. This difference in resolution originates from the sub-diffusive spatial frequency dependence of $\gamma$, with spatial resolution $<1$ mean free path, while $\mu_{\mathrm{s}}^{\prime}$ has both diffusive and sub-diffusive spatial frequency dependence resulting in a resolution $\approx 1-2$ mean free paths. Sensitivity to changes in optical properties is given by the line spread function in Figure S3(f), which is the derivative of the edge response function. The maximum change in recovered optical property between adjacent pixels is 0.09 for $\gamma$ and $0.22 \mathrm{~mm}^{-1}$ for $\mu_{\mathrm{s}}^{\prime}$.

\section{Dark Field Imaging of Ex-Vivo Tissue Samples}

Each of the tissue specimens was imaged with the spatial frequency imaging technique as shown in Figure 2, while a select set of specimens were also imaged with a dark-field reflectance microscope. The details of the dark-field microscope setup is described elsewhere [13]. The illumination and bandwidth for the dark-field imaging were selected by a home-built LabView program to take a series of single shot images, one wavelength at a time, with wavelength scanned from $450 \mathrm{~nm}$ to $650 \mathrm{~nm}$ with a $10 \mathrm{~nm}$ bandwidth. Only a limited set of images were acquired for a selected sample area, as it would take roughly $20 \mathrm{~min}$ to scan the entire specimen by patch-wise imaging to cover the entire sample and put the patches together. The origin of the dark-field signal is inherently related to localized backscattering; hence, it provided a unique standard with which to compare the structured light scatter signal. As with structured light imaging, dark field microscopy imaging was performed through a glass slide to minimize surface curvature effects. 


\section{E. Selection of Colormaps for Optical Parameter Visualization}

The selection of color maps used to display maps of optical properties were chosen as follows. For $\gamma, \mu_{\mathrm{s}}^{\prime}$, and scatter slope, fully saturated HSV color maps were used, with a saturation and brightness value of one. Each of these color maps goes through half of the hue spectrum, with the $\gamma$ color map spanning red, yellow, and green and the $\mu_{\mathrm{s}}^{\prime}$ color map spanning cyan, blue, and magenta. The range of the color bar axes in each figure was different, as the range of scattering values in the phantom sets versus the tissue is different. However, the range of the color bar axes within each figure, most notably in Figure 5, is the same in order to provide direct visual comparison between the tissue types. For the $\mu_{\mathrm{a}}$ map in Figure S1(b), the default MATLAB, 2015a "parula" map was used.

\section{REFERENCES}

1. N. Bodenschatz, A. Brandes, A. Liemert, and A. Kienle, "Sources of errors in spatial frequency domain imaging of scattering media," Journal of biomedical optics 19, 071405071405 (2014).

2. R. Michels, F. Foschum, and A. Kienle, “Optical properties of fat emulsions," Optics Express 16, 5907-5925 (2008).

3. S. C. Kanick, D. M. McClatchy, V. Krishnaswamy, J. T. Elliott, K. D. Paulsen, and B. W. Pogue, "Sub-diffusive scattering parameter maps recovered using wide-field high-frequency structured light imaging," Biomedical optics express 5, 33763390 (2014).

4. D. J. Cuccia, F. Bevilacqua, A. J. Durkin, F. R. Ayers, and B. J. Tromberg, "Quantitation and mapping of tissue optical properties using modulated imaging," Journal of biomedical optics 14, 024012-024012 (2009).

5. V. Krishnaswamy, J. T. Elliott, D. M. McClatchy, R. J. Barth, W. A. Wells, B. W. Pogue, and K. D. Paulsen, "Structured light scatteroscopy," Journal of biomedical optics 19, 070504070504 (2014).

6. S. L. Jacques, “Optical properties of biological tissues: a review," Physics in medicine and biology 58, R37 (2013).

7. R. C. Haskell, L. O. Svaasand, T.-T. Tsay, T.-C. Feng, B. J. Tromberg, and M. S. McAdams, "Boundary conditions for the diffusion equation in radiative transfer," JOSA A 11, 2727-2741 (1994).

8. F. Bevilacqua, D. Piguet, P. Marquet, J. D. Gross, B. J. Tromberg, and C. Depeursinge, "In vivo local determination of tissue optical properties: applications to human brain," Applied optics 38, 4939-4950 (1999).

9. F. van Leeuwen-van Zaane, U. Gamm, P. van Driel, T. Snoeks, H. de Bruijn, A. van der Ploeg-van den Heuvel, I. Mol, C. Löwik, H. Sterenborg, A. Amelink, and D. Robinson, "In vivo quantification of the scattering properties of tissue using multi-diameter single fiber reflectance spectroscopy," Biomedical optics express 4, 696-708 (2013).

10. A. J. Radosevich, A. Eshein, and V. Backman, "Subdiffusion reflectance spectroscopy to measure tissue ultrastructure and microvasculature: model and inverse algorithm," Journal of biomedical optics 20, 097002-097002 (2015).

11. F. Bevilacqua and C. Depeursinge, "Monte carlo study of diffuse reflectance at source-detector separations close to one transport mean free path," JOSA A 16, 2935-2945 (1999).

12. S. D. Konecky, T. Rice, A. J. Durkin, and B. J. Tromberg, "Imaging scattering orientation with spatial frequency domain imaging," Journal of biomedical optics 16, 1260011260018 (2011).
13. A. Ray, R. Kopelman, B. Chon, K. Briggman, and J. Hwang, "Scattering based hyperspectral imaging of plasmonic nanoplate clusters towards biomedical applications," Journal of biophotonics (2015). 